\title{
First-Principles Thermodynamics of Coherent Interfaces in Samarium-Doped Ceria Nanoscale Superlattices
}

\author{
A. van de Walle* \\ Engineering and Applied Science Division, California Institute of Technology, Pasadena, California 91125, USA
}

D. E. Ellis ${ }^{\dagger}$

Chemistry Department, Northwestern University, Evanston, Illinois 60208, USA

(Received 7 March 2007; published 27 June 2007)

\begin{abstract}
Nanoscale superlattices of samarium-doped ceria layers with varying doping levels have been recently proposed as a novel fuel cell electrolyte. We calculate the equilibrium composition profile across the coherent $\{100\}$ interfaces present in this system using lattice-gas Monte Carlo simulations with long-range interactions determined from electrostatics and short-range interactions obtained from $a b$ initio calculations. These simulations reveal the formation of a diffuse, nonmonotonic, and surprisingly wide $(11 \mathrm{~nm}$ at $400 \mathrm{~K}$ ) interface composition profile, despite the absence of space charge regions.
\end{abstract}

DOI: 10.1103/PhysRevLett.98.266101

PACS numbers: 68.35.- p, 02.70.Uu, 05.70.Np, 71.15.Mb

The performance of solid oxide fuel cells [1] is steadily increasing, thanks to the development of novel electrode and electrolyte materials. A recent trend is the use of nanostructured materials to devise electrolytes and electrodes with improved properties [2]. In particular, nanoscale superlattices consisting of layers of the samariumdoped ceria ion conductor with various levels of doping have been proposed as an avenue to improve ionic conductivities of the electrolyte in the low-temperature regime $[3,4]$. Optimizing this approach as well as understanding the mechanisms underlying the increased ionic conductivity requires a detailed understanding of the interfacial properties in this system.

In this Letter, we present $a b$ initio calculations of the equilibrium composition profile (of dopants and vacancies) across an array of coherent $\{100\}$ interfaces mimicking the experimentally synthesized samarium-doped ceria superlattices. These calculations reveal two surprising findings. First, the coherent interface width is large and represents a significant fraction of the period of superlattices $[3,4]$, thus suggesting a very plausible explanation for the observed significant ionic conductivity improvements. Interestingly, this phenomenon is not driven by space charge effects $[5,6]$, the most commonly invoked explanation for longrange deviations from the bulk equilibrium vacancy composition. The composition profile also exhibits composition oscillations, a feature that, to our knowledge, has never been noted in ceramic coherent interfaces.

The determination of the equilibrium $\{100\}$ interface composition profile in this system demands simulation cell sizes that are beyond the reach of a brute-force $a b$ initio approach: In order to obtain results that are converged with respect to system size, a system consisting of over 70000 atoms is needed. Moreover, finding the equilibrium profile requires extensive sampling of configuration space that would be prohibitive via ab initio methods alone. To address these issues, we rely on the cluster expansion formalism [7-10], which represents the energy $E$ of a crystalline alloy with a computationally efficient Hamiltonian taking the form of a polynomial in terms of occupation variables $\sigma_{i}$ indicating the type of atom residing on each lattice site $i$ :

$$
E=\sum_{i \neq j} J_{i j} \sigma_{i} \sigma_{j}+\sum_{i \neq j \neq k} J_{i j k} \sigma_{i} \sigma_{j} \sigma_{k}+\cdots .
$$

The unknown coefficients $J \ldots$ of this polynomial are called effective cluster interactions (ECI) and are fit to a database of $a b$ initio structural energies. It has been formally shown that an infinite series of this form can represent any configuration dependence of the energy [7]. Moreover, formal methods have been developed to determine the number of terms and the database size needed to achieve a given precision [11]. The ECI can typically be determined from reasonably sized database of $a b$ initio calculations involving small-unit-cell atomic arrangements. These ECI can then be used in large-scale equilib-
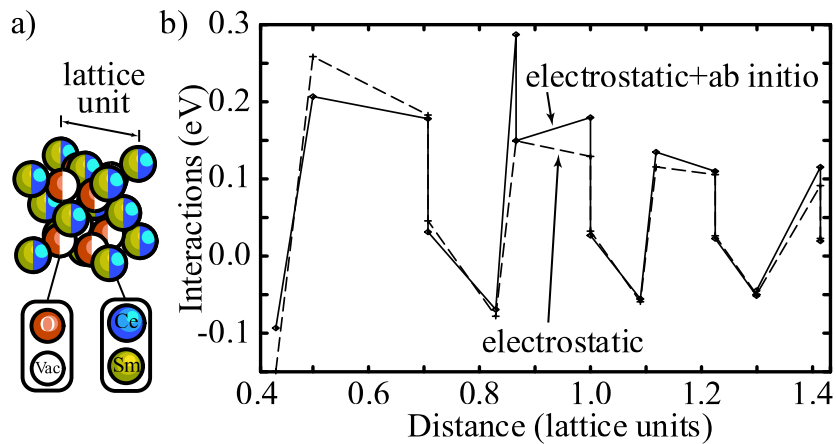

FIG. 1 (color online). (a) Coupled-sublattice lattice-gas model describing the samarium-doped ceria system on the fluorite lattice. (b) Effective cluster interactions $(J \ldots)$. 
rium lattice-gas Monte Carlo (MC) simulations of the interface, without necessitating large-scale $a b$ initio calculations.

Because of the dual-sublattice nature of the doped ceria system [see Fig. 1(a)], we employ a generalization of the cluster expansion designed to handle coupled sublattices [12]. In addition, the infinite-range electrostatic interactions are explicitly taken into account. The difference between the total and the electrostatic energies is represented via a short-range cluster expansion. The prefactor in the Coulomb potential is adjusted so as to obtain the shortest-range ECI possible. This approach allows for deviation from the vacuum permittivity, which can arise from the screening of the charges via polarizability of intervening electronic shells (reflecting the macroscopic dielectric constant of the material) or from the finite size of each atom's charge distribution. The fitted prefactor in the Coulomb potential is 20 smaller than its value in vacuum (corresponding to a relative permittivity of 20). In the Monte Carlo simulations, the electrostatic energy is efficiently calculated via reciprocal-space summations using the updating algorithm described in Ref. [13].

Long-range elastic interactions can also be explicitly included in the cluster expansion using a reciprocal-space representation of the so-called constituent strain energy [14]. While elastic interactions are important to take into account in interfacial thermodynamics studies when the atomic size mismatch is large [15], we do not include these contributions here, because the lattice mismatch between the two phases we consider is very small (less than $0.4 \%$ ) and because we do not see any evidence of residual longrange interactions once the long-range electrostatic contributions are subtracted.

Our database of 71 structures contains 61 structures that are "charge-balanced" (i.e., they have a charge of zero if all species take their nominal charges of $+4,+3$, and -2 for $\mathrm{Ce}, \mathrm{Sm}$, and $\mathrm{O}$, respectively). This oversampling improves accuracy in the region that will be mostly sampled in the Monte Carlo simulations. A few "off-chargebalance" structures (10) are also included to ensure that the ECI can accurately model the energetics of the system even when the system locally fluctuates away from perfect charge balance. Of course, the term off-charge-balance structure is a misnomer, since deviations from the nominal charges due to charge transfer necessarily take place to maintain overall charge balance (in reality and in the ab initio calculations).

Figure 1(b) shows the decomposition of the effective cluster interactions into electrostatic and nonelectrostatic components. Although the nonelectrostatic corrections appear small in magnitude, they cannot simply be neglected. They are responsible for stabilizing the ordered compounds that form in this system at low temperatures and control the order-disorder transition temperatures for each of these ordered compounds. The fact that all interactions

are pairwise is not an imposed approximation. The terms to include in the cluster expansion were selected according to the cross-validation criterion [11], which showed that including three-body, four-body, etc., interactions did not significantly improve the predictive power of the cluster expansion.

The $a b$ initio calculations were performed with the VASP package [16] using the projector augmented wave method, while all of the remaining steps (structural database generation, fit of the cluster expansion, and lattice-gas Monte Carlo simulations) were carried out with the Alloys Theoretic Automated Toolkit [11,17-19]. The parameters controlling the accuracy of the simulations are given in Table I.

The cluster expansion and Monte Carlo simulations first enable us to investigate the phase equilibria in this system. It is well established that the oxygen-vacancy sublattice undergoes a phase separation at high oxygen content. In pure $\mathrm{CeO}_{y}$, this miscibility gap is centered around $y=$ 1.93 [20], while in $\mathrm{Ce}_{0.818} \mathrm{Sm}_{0.182} \mathrm{O}_{y}$, it is centered around $y=1.79$ [21]. Our calculations confirm the presence of this miscibility gap and further predict that, in thermodynamic equilibrium, phase separation should take place on the cation sublattice as well. We predict that this miscibility gap closes at around $1000 \mathrm{~K}$. This miscibility gap on the cation sublattice has apparently not been noted in experimental investigations, perhaps due to the slower diffusion kinetics on the cation sublattice associated with the absence of vacancies. Nevertheless, our results suggest that, after prolonged operation at high temperature, phase separation should occur in the electrolyte. It is physically plausible that the oxygen sublattice phase separation would drive a cation sublattice phase separation, as this would allow all species to regain their nominal charge and to reduce the electrostatic energy of the system.

TABLE I. Calculation parameters. The convention used to describe the $k$-point mesh density is described in Ref. [18]. The cross-validation score measures the predictive power of the cluster expansion and is defined in Ref. [11]. The supercell size is given in multiples of the conventional cubic unit cell.

\section{$A b$ initio calculations}

Energy cutoff

$k$-point mesh density

Exchange-correlation functional

Cluster expansion construction

Number of structures in database

Number of clusters in cluster expansion

Cross-validation score

Monte Carlo simulations

Equilibration MC passes

MC passes between interface snapshots

Supercell size
$400 \mathrm{eV}$

$1000 /\left(\right.$ atom $\left.^{-1}\right)$ PW91

71

20

$0.07 \mathrm{eV}$

75

25

$8 \times 8 \times 96$ 
Our simulations model an equilibrium between the disordered solid solutions at each side of this miscibility gap at $400 \mathrm{~K}$, namely, $\mathrm{Ce}_{0.54} \mathrm{Sm}_{0.46} \mathrm{O}_{1.77}$ and $\mathrm{Ce}_{0.95} \mathrm{Sm}_{0.05} \mathrm{O}_{1.975}$. We consider an oxygen content such that the system is "charge-balanced" when the Ce, Sm, and $\mathrm{O}$ species are assigned nominal charges of $+4,+3$, and -2 , respectively. We used a relatively low temperature in order to obtain an interface sufficiently narrow to be fully contained in a unit cell of a manageable size. Partially converged simulations at higher temperatures indicate that the interface is even wider between 400 and $1000 \mathrm{~K}$, although precisely quantifying by how much would have required significantly more computational resources. Nevertheless, even at $400 \mathrm{~K}$, the interface is already sufficiently wide to enable us to conclude that most of the volume of the superlattice structure would have a vacancy concentration deviating from the bulk equilibrium values. The interface energy [22] at $400 \mathrm{~K}$ is $300 \pm 80 \mathrm{~mJ} / \mathrm{m}^{2}$, which is a fairly typical value for a solid-solid coherent interface [15,23].

Our calculated equilibrium composition profiles (see Fig. 2) exhibit two important characteristics. First, the $10 \%-90 \%$ width of the interface (i.e., the region where the composition deviates from the equilibrium bulk values by more than 10\%) is $11 \mathrm{~nm}$, which is very large. It is, for instance, much larger than the typical diffuse coherent interface in metal alloys (at most a few nanometers) $[15,24,25]$. Since the period of the superlattices described in Refs. [3,4] is of the order of $50 \mathrm{~nm}$, a large fraction (about 0.44 ) of the volume of the electrolyte has a vacancy composition which is significantly affected by the interfa-

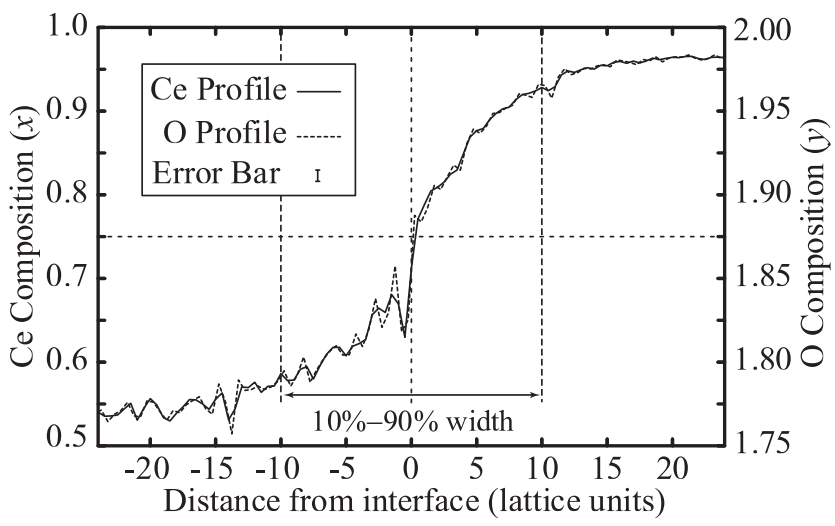

FIG. 2. Equilibrium composition profile. The solid line (with the ordinate on the left axis) gives $x$ in the formula $\mathrm{Ce}_{x} \mathrm{Sm}_{1-x} \mathrm{O}_{y}$, while the dotted line (with the ordinate on the right axis) gives $y$. The vertical scale of each curve is selected such that, when the two curves overlap, there is no net charge in that region in space. The profiles represent average compositions from 160 snapshots from the Monte Carlo simulations. The error bar represents $2 \sigma$ confidence intervals for the mean composition (nearly identical for $\mathrm{Ce}$ and $\mathrm{O}$ on the scales of the curves). One lattice unit is $5.44 \AA$ A. The horizontal range of the plot covers half the period of the superlattice. ces. This large diffuse interface width is not driven by the formation of a space charge: As seen in Fig. 2, the absolute values of the anion and cation contributions to the charge profile are nearly indistinguishable within the simulation noise. Note that this agreement is not an artifact of imposing charge neutrality of the whole system. Local deviations from charge neutrality are allowed but just happen to be very energetically costly in this system and are therefore very small in equilibrium.

Our situation is different from the well-known case where a net charge is trapped at a grain boundary $[6,26]$ and screened by a region where the vacancy composition differs from the bulk equilibrium value. Here we have a coherent interface, and there is therefore nothing structurally different at the interface that could trap charge-all charges are free to move.

Interestingly, the interface brings the composition of the electrolyte close to the known optimal doping level corresponding to a composition of $\mathrm{Ce}_{0.818} \mathrm{Sm}_{0.182} \mathrm{O}_{1.909}[27,28]$. Otherwise, the optimal doping level lies in the middle of a miscibility gap, indicating that, although alloys with such composition can be synthesized, they run the risk of phaseseparating after long term and high-temperature operation. In contrast, a superlattice structure locks in a composition profile that is close to optimal throughout most of the volume. While it can be argued that the true equilibrium state of the system would be a macroscopically phaseseparated system, this equilibrium appears difficult to reach from a superlattice-type initial state.

The second interesting feature of the composition profile is its nonmonotone nature. Clear oscillations in the profile can be seen near the interface, a feature that, to our knowledge, has never been noted in ceramic coherent interfaces. However, density oscillations are well known in other systems dominated by electrostatic interactions, such as liquid alkali metal surfaces $[29,30]$. It has been clearly shown in these systems that such oscillations can arise when the free energy $F$ of the system takes a general density-functional form (adapted from Ref. [30]):

$$
F=G\left[\rho_{+}, \rho_{-}\right]+\int\left[\rho_{+}(r)-\rho_{-}(r)\right] \phi(r) d r,
$$

where $\rho_{+}(r)$ and $\rho_{-}(r)$ are the densities of cation and anion, respectively, $G[]$ is a free energy functional of the cation and anion densities, and $\phi(r)$ is the electrostatic potential (self-consistently determined from the densities). The free energy of the Sm-doped ceria system can be cast in this form as well, the only difference being that the "densities" would be defined on a discrete lattice rather than on a continuum. The functional $G[]$ could, in principle, be determined from our cluster expansion Hamiltonian via Monte Carlo thermodynamic integration, although this is not needed for our argument. Given this analogy, the elegant perturbative asymptotic approximation presented in Ref. [30] can be used to obtain the equilibrium profile minimizing Eq. (1) as the solution to a system of three 
coupled second-order differential equations in the densities $\rho_{+}(r)$ and $\rho_{-}(r)$ and the potential $\phi(r)$. Under a wide range of conditions, this system admits damped oscillatory solutions. In contrast, without electrostatic interactions, a similar perturbative treatment simply leads to CahnHilliard-type equations [31] whose solution is necessarily monotone, as observed, for instance, in solid-state metallic alloys.

In summary, large-scale Monte Carlo simulations with interaction parameters derived from $a b$ initio calculations were used to determine the equilibrium composition profile across a coherent interface in samarium-doped ceria superlattices. We find that no space charge region forms and that the coherent interfaces are diffuse with a width of about $11 \mathrm{~nm}$. This allows the system to reach close to the optimal dopant concentration over wide regions, even though this optimal doping level lies in the middle of a miscibility gap. We find composition oscillations near the interface that have never been reported before in this type of system. Similar oscillations have been observed in other systems with strong electrostatic interactions (i.e., alkali metals), and we propose that a common underlying mechanism is at work.

This material is based upon work supported by the Department of Energy under Grant No. DE-FG0205ER46255. This research was supported in part by the National Science Foundation through TeraGrid resources provided by SDSC and NCSA under Grants No. DMR050013N and No. DMR070025T.

*avdw@alum.mit.edu

†dvmgroup@gmail.com

[1] S.A.I.C. EG\&G Services Parsons, Inc., Fuel Cell Handbook(U.S. Department of Energy, Washington, DC, 2000), http://www.fuelcells.org/info/library/fchandbook.pdf.

[2] E. B. Lavnik, I. Kosacki, H. L. Tuller, and J. Y. Ying, J. Electroceram. 1, 205 (1997); A. Tschöpe, J. Y. Ying, and H. L. Tuller, Sens. Actuators B, Chem. 31, 111 (1996); J. Hwang and T. O. Mason, Z. Phys. Chem. (Frankfort/ Main) 207, 21 (1998); A. Tschöpe, E. Sommer, and R. Birringer, Solid State Ionics 139, 255 (2001); T. Suzuki, I. Kosacki, and H. U. Anderson, J. Am. Ceram. Soc. 85, 1492 (2002); I. Kosacki, V. Petrovsky, H. U. Anderson and P. Colomban, J. Am. Ceram. Soc. 85, 2646 (2002); T. Suzuki, I. Kosacki, and H. U. Anderson, Solid State Ionics 151, 111 (2002); I. Kosacki, H. U. Anderson, Y. Mizutani, and K. Ukai, Solid State Ionics 152-153, 431 (2002).

[3] I. Kosacki, C. M. Rouleau, P. F. Becher, and D. Lowndes (to be published).

[4] I. Kosacki, C. M. Rouleau, P. F. Becher, J. Bentley, and D. H. Lowndes, Solid State Ionics 176, 1319 (2005).

[5] K. L. Kliewer and J. A. Koehler, Phys. Rev. 140, A1226 (1965).
[6] A. Tschöpe, C. Bäuerle, and R. Birringer, J. Appl. Phys. 95, 1203 (2004).

[7] J. M. Sanchez, F. Ducastelle, and D. Gratias, Physica (Amsterdam) 128A, 334 (1984).

[8] D. de Fontaine, in Solid State Physics, edited by H. Ehrenreich, F. Seitz, and D. Turnbull (Academic, New York, 1994), Vol. 47, p. 33.

[9] A. Zunger, in Statics and Dynamics of Alloy Phase Transformation, edited by P.E. Turchi and A. Gonis, NATO ASI Vol. 319 (Plenum, New York, 1994), p. 361.

[10] F. Ducastelle, Order and Phase Stability in Alloys (Elsevier Science, New York, 1991).

[11] A. van de Walle and G. Ceder, J. Phase Equilib. 23, 348 (2002).

[12] P. D. Tepesch, G. D. Garbulsky, and G. Ceder, Phys. Rev. Lett. 74, 2272 (1995).

[13] Z. W. Lu, D. B. Laks, S.-H. Wei, and A. Zunger, Phys. Rev. B 50, 6642 (1994).

[14] D. B. Laks, L. G. Ferreira, S. Froyen, and A. Zunger, Phys. Rev. B 46, 12587 (1992).

[15] M. Asta, S. M. Foiles, and A. A. Quong, Phys. Rev. B 57, 11265 (1998).

[16] G. Kresse and J. Furthmüller, Phys. Rev. B 54, 11169 (1996); G. Kresse and J. Furthmüller, Comput. Mater. Sci. 6, 15 (1996).

[17] A. van de Walle and M. Asta, Model. Simul. Mater. Sci. Eng. 10, 521 (2002).

[18] A. van de Walle, M. Asta, and G. Ceder, CALPHAD: Comput. Coupling Phase Diagrams Thermochem. 26, 539 (2002).

[19] A. van de Walle, The Alloy Theoretic Automated Toolkit, 2001, http://www.its.caltech.edu/ avdw/atat/.

[20] P. Subramanian, in Binary Alloy Phase Diagrams, edited by T. Massalski (ASM International, Materials Park, $\mathrm{OH}$, 1990), Vol. II, p. 1089.

[21] N. Stelzer, J. Nölting, and I. Riess, J. Solid State Chem. 117, 392 (1995).

[22] This number was obtained from $\left[E_{8 \times 8 \times 80}-\right.$ $\left.(80 / 96) E_{8 \times 8 \times 96}\right] /[1-(80 / 96)] /(2 A)$, where $E_{h \times k \times l}$ is the total energy of a $h \times k \times l$ supercell containing two (001) interfaces of area $A$.

[23] A. van de Walle and M. Asta, Metall. Mater. Trans. A 33A, 735 (2002).

[24] M. Asta and J. J. Hoyt, Acta Mater. 48, 1089 (2000).

[25] C. K. Sudbrack, K.E. Yoon, R.D. Noebe, and D. N. Seidman, Acta Mater. 54, 3199 (2006).

[26] X. Guo and R. Waser, Solid State Ionics 173, 63 (2004).

[27] H. Yahiro, Y. Eguchi, K. Eguchi, and H. Arai, J. Appl. Electrochem. 18, 527 (1988).

[28] H. Inaba and H. Tagawa, Solid State Ionics 83, 1 (1996).

[29] J. Goodisman and M.-L. Rosinberg, J. Phys. C 16, 1143 (1983).

[30] M. Iwamatsu and S. K. Lai, J. Phys. Condens. Matter 4, 6039 (1992).

[31] J. W. Cahn and J.E. Hilliard, J. Chem. Phys. 28, 258 (1958). 